\title{
Dynamique de colonisation de l'aulne glutineux Alnus glutinosa (L) dans un marais eutrophe de la plaine alluviale du Rhône *
}

\author{
C Cluzeau ** \\ Université Joseph Fourier, laboratoire d'écologie végétale, BP 53 X, 38401 Grenoble Cedex; \\ Université Claude Bernard, laboratoire de biologie animale et écologie, Lyon I, \\ 43 bd du 11 novembre 1918, 69622 Villeurbanne Cedex, France
}

(Reçu le 20 mars 1992; accepté le 11 mai 1992)

\begin{abstract}
Résumé - Les premières formations boisées s'installant dans les marais eutrophes lors du processus naturel d'atterrissement, sont des formations hygrophiles de type saussaie à Salix cinerea ou aulnaie à Alnus glutinosa. Ces essences pionnières, grâce à leur capacité de dispersion, colonisent rapidement les formations herbacées auxquelles elles succèdent. Afin de décrire la dynamique de colonisation de l'aulne glutineux dans le marais de Lavours, nous avons étudié la distribution spatiale des circonférences à l'intérieur d'une population échantillon. L'analyse factorielle des correspondances réalisée sur les distributions des circonférences met en évidence une succession de vagues de colonisation. Chaque vague de colonisation correspond à une progression de l'aulne de 100$150 \mathrm{~m}$ vers l'intérieur du marais. La période séparant 2 vagues est de l'ordre de 15 ans.
\end{abstract}

marais eutrophe / colonisation / Alnus glutinosa / classe de circonférence / analyse factorielle des correspondances

Summary - Colonization dynamics of alder (Alnus glutinosa) in an eutrophic swamp of the floodplain of the upper Rhone. The establishment of alder (Alnus glutinosa) in eutrophic swamps is a part of the natural pattern of plant succession. Its abundant wind- and water-dispersed seeds allow it to colonize early successional sites with herbaceous species such as Phragmites sp or Carex sp. In order to describe the colonization dynamics of alder (Alnus glutinosa) in the Lavours' swamp, we studied the spatial distribution of the circumference. At each sample point, the circumference of the 30-50 trees which were located around the point were measured. Data analysis consisted of an ordination of the circumference distributions using a factorial correspondence analysis. The results showed that invasion occurred in a stepwise fashion. The spread of alder is determined by

\footnotetext{
* Ce travail a été réalisé dans le cadre du programme interdisciplinaire de recherche sur l'environnement (PIREN) du CNRS.

** Adresse actuelle : INRA, centre de recherche forestière de Nancy, station de sylviculture et de production, Champenoux, F-54280 Seichamps, France.
} 
the mean distance of seed dispersion and the age at maturity. In the plot studied, alder takes 15 years to cover $100-150 \mathrm{~m}$, but the speed of extension probably varies with environmental factors and human disturbance.

eutrophic swamp / colonization / Alnus glutinosa / size-classes / factorial correspondence analysis

\section{INTRODUCTION}

Les marais sont des zones dépressionnaires humides, en cours de colmatage. Dans les plaines alluviales, ils sont généralement isolés du chenal principal et ne sont donc plus soumis directement à l'influence des crues (Pautou, 1980; Pautou et al, 1985b). Seul l'affleurement périodique de la nappe assure la pérennité de groupements végétaux aquatiques et semi-aquatiques constitués d'hydrophytes, qui progressivement vont transformer leur biotope dans le sens d'un atterrissement.

Les peuplements d'hélophytes du genre Phragmites ou Carex, caractérisés par une forte évapotranspiration et une forte production de biomasse, contribuent à l'assèchement et à l'exhaussement des sols. $\mathrm{Au}$ fur et à mesure de l'enfoncement de la nappe, des formations végétales se succèdent depuis les groupements semiaquatiques jusqu'à l'apparition de forêts hygrophiles : type saussaie à Salix cinerea ou aulnaie à Alnus glutinosa, puis mésohygrophiles à mésophiles : type ormaje à UImus minor ou chênaie-frênaie à Quercus robur (Pautou et al, 1979; Pautou et al, 1985a). L'installation de la formation boisée correspond au stade ultime de l'évolution des milieux semi-humides.

Les marais ont longtemps été cultivés par l'homme. Les fauches régulières et le pâturage ont permis le maintien des prairies humides, en s'opposant au dynamisme naturel de la végétation. Depuis la fin du XIXe siècle, l'abandon progressif des pratiques culturales dans les zones marécageuses a favorisé l'extension des ligneux à partir de semenciers (arbres matures) déjà en place. Cette colonisation, bien que s'inscrivant dans le processus d'atterrissement, est accélérée par les opérations de drainage et les modifications de mise en eau provoquées par les aménagements hydroélectriques (Roux et al, 1982; Pautou et Decamps, 1985). Ainsi, la progression des aulnes dans le marais est fortement liée au réseau de fossés naturels et artificiels, creusés au XIX siècle pour drainer les eaux stagnantes et faciliter l'extraction de la tourbe (Bravard, 1987). Les aulnes les plus âgés sont concentrés le long des rivières et des fossés car la dissémination des graines par l'eau courante est très efficace et car ces zones ont été épargnées par les pratiques culturales. Ces aulnaies riveraines constituent des foyers de propagation, grâce à l'entraînement des graines par les crues et à leur accumulation dans les dépressions marginales (Lhote, 1985).

Le marais de Lavours (Ain), situé dans la plaine alluviale du Haut-Rhône à proximité du lac du Bourget, est classé réserve naturelle depuis 1984. La progression particulièrement rapide de l'aulne glutineux pose des problèmes aux gestionnaires de la réserve car l'augmentation des surfaces boisées a un effet d'homogénéisation sur la flore et la faune et entraîne une régression des espaces semi-aquatiques riches en espèces remarquables (Pautou, 1985).

Afin de décrire la dynamique d'extension de cette essence, nous avons étudié 
la distribution spatiale des circonférences dans un secteur du marais fortement colonisé.

\section{MATÉRIEL, SITE ET MÉTHODES}

\section{Site d'étude}

Le marais de Lavours se situe dans l'hydrosystème du Haut-Rhône français. C'est une dépression de forme triangulaire bordée au nord par la montagne du Grand-Colombier (extrémité méri- dionale du Jura), à l'ouest par la rivière le Séran et à l'est par le Rhône et son bourrelet alluvial (Sogreah, 1960; Ain et Pautou, 1969) (fig 1). II est entouré d'une ceinture boisée, en majorité constituée d'aulnes glutineux, qui longe les cours d'eau et gagne vers l'intérieur du marais.

Le secteur d'étude occupe une surface de 9 ha, dans une zone du marais où l'aulne est en pleine expansion (fig 1). La partie est de ce secteur (2 ha) est close et sert de réserve pour une expérience sur le contrôle de la végétation par des vaches rustiques écossaises (Highland Cattles) et des chevaux camarguais (Manneville et Majchrzak, 1988). Cet enclos est entouré d'un pare-feu qui a été fauché pendant l'été 1987.

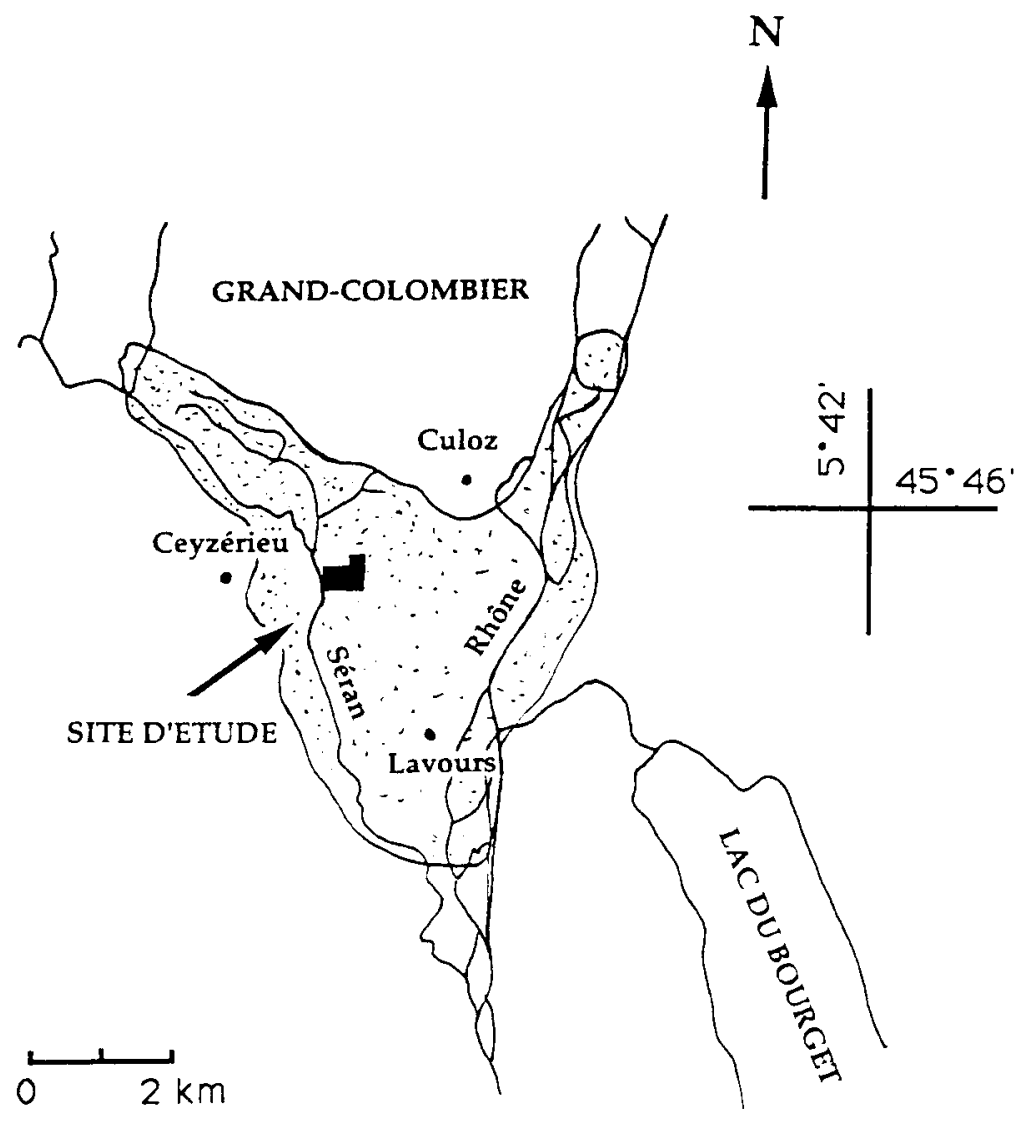

Fig 1. Localisation du secteur d'étude. 


\section{Méthodes}

L'aulne glutineux est une essence héliophile dont la régénération est rarement observée sous couvert forestier car les semis ont besoin de lumière pour s'installer (Mac Vean, 1953). Son extension est donc particulièrement favorisée lorsqu'une zone ouverte se trouve à proximité d'un bois ou d'un bosquet (Vinther, 1983; Olsson, 1987).

Ce schéma de colonisation a été décrit par Harper (1977), en prenant pour exemple le pin Pinus taeda. Cette essence pionnière du sudest des États-Unis envahit les prairies abandonnées par vagues de colonisation successives, chaque étape correspondant à la période nécessaire pour que les graines dispersées par les semenciers donnent des individus matures qui deviennent semenciers à leur tour. La distance parcourue par le front de progression dé- pend des capacités de dispersion de l'espèce. Partant de ce schéma pour l'aulne, l'objectif de notre étude est d'individualiser et de caractériser les vagues de colonisation.

L'observation en stéréoscopie des photographies aériennes prises dans le secteur d'étude met en évidence une succession dans l'espace de groupes d'aulnes de hauteur identique, décroissante de l'ouest vers l'est. Afin de confirmer cette structure et de définir les limites spatiales de ces groupes, nous avons cartographié les circonférences des arbres grâce à un échantillonnage systématique. Notre choix s'est porté sur les circonférences car c'est une mesure plus simple et plus rapide à réaliser que celle des hauteurs.

Nous avons réalisé 2 séries de mesures à partir d'un quadrillage de maille carrée de $40 \mathrm{~m}$ de côté (fig 2). Ce pas a été choisi d'après les photographies aériennes, de façon à prospecter

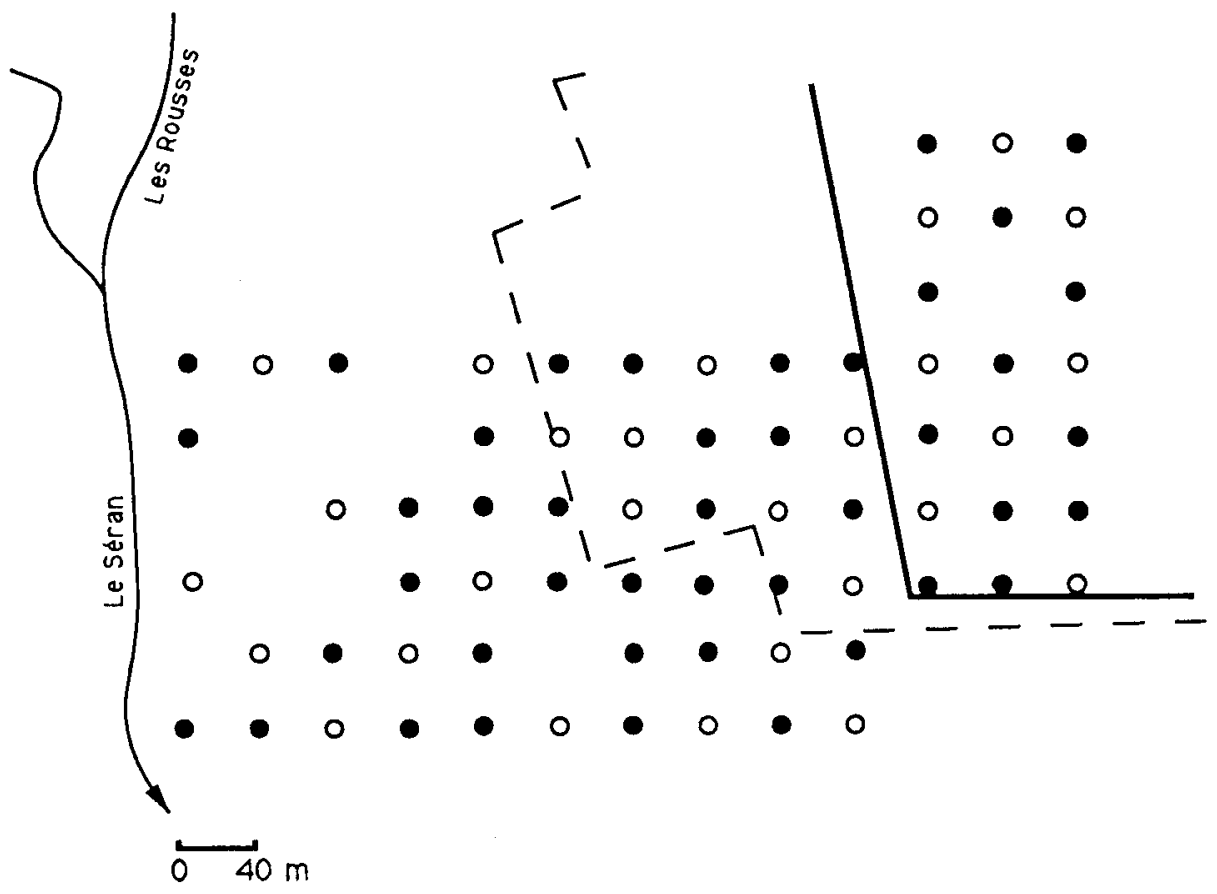

Fig 2. Disposition spatiale des relevés dans le secteur d'étude. Chaque point-relevé est situé à un nœud du quadrillage (maille $40 \times 40 \mathrm{~m}$ ). Ont été supprimés ceux qui se trouvaient sur un chemin ou en lisière de la forêt. - Points-relevés de la première série de mesures $(n=69)$; points-relevés ayant été retenus pour la deuxième série de mesures $(n=27)$. - Limite ouest de l'enclos; --- limite ouest de la zone fauchée (pare-feu). 
tous les groupes de hauteur visibles. Chaque nœud du quadrillage est matérialisé sur le terrain par un piquet et fait l'objet d'un relevé. Soixante neuf points ont été retenus sur les 81 initiaux. Ont été supprimés ceux qui se trouvaient dans les trouées, les fossés ou les chemins.

Pour le premier échantillonnage, nous avons utilisé la méthode des "quadrants centrés sur un point" ou "point-centered quadrat method" de Cottam et Curtis (1956) (Pielou, 1959; Gounot, 1969; Bergeron et Dubuc, 1989). Chaque relevé est effectué de la façon suivante : l'espace entourant le piquet est divisé en 4 quarts égaux, ou quadrants, dans lesquels la distance à l'arbre le plus proche du piquet est mesurée ainsi que la circonférence de cet arbre (fig 3 ). Lorsque les arbres ont une circonférence inférieure à $5 \mathrm{~cm}$, on mesure alors leur hauteur. Grâce au quadrillage, chaque relevé est repéré par ses coordonnées spatiales. II est donc possible d'établir une carte des circonférences moyennes et des densités.

$\mathrm{Au}$ vu de ces premiers résultats, un deuxième échantillonnage a été réalisé. Étant donné l'hétérogénéité des circonférences dans la partie ouest du secteur, la moyenne réalisée sur 4 individus nous paraîssait peu représentative. Vingt-sept relevés répartis de façon régulière sur le quadrillage ont donc fait l'objet d'un deuxième échantillonnage prenant en compte

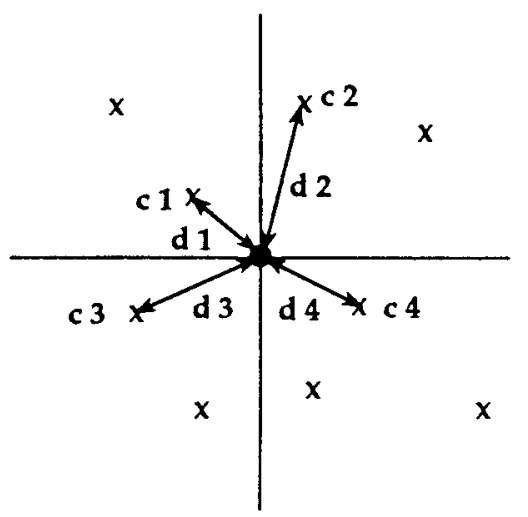

Fig 3. Schéma expliquant la méthode des quadrants centrés sur un point de Cottam et Curtis (1956). - piquet matérialisant un relevé; - limite des quadrants; $X$ arbre; di distance à l'arbre le plus proche du piquet; ci circonférence de cet arbre. les 30-50 individus (selon la densité) les plus proches du piquet (fig 2). Les données se présentent alors sous forme d'histogrammes en classes de circontérences et sont traitées par une analyse factorielle des correspondances (AFC) afin de faire apparaître une typologie basée sur la distribution des circonférences et non sur la moyenne. Cette méthode d'ordination est détaillée dans Persat et Chessel (1989).

Les analyses statistiques et les représentations graphiques ont été réalisées sur Macintosh grâce aux logiciels "Macmul" et "Graphmu" (Thioulouse, 1989).

\section{RÉSULTATS}

\section{Première série de mesures}

Chaque point du quadrillage est caractérisé par la moyenne des circonférences des 4 arbres mesurés et par une estimation de la densité $d$ (Cottam et Curtis, 1956).

$d=1 / M ; M$ «mean area» correspond à la surface occupée par un arbre (en m) et se calcule de la façon suivante :

soit $x_{i}$ la valeur de la distance (en $m$ ) séparant l'arbre i du piquet, $1<i<4: \Sigma x_{i} / 4=$ $\sqrt{M}$

Le secteur étudié présente une phase récente d'extension de l'aulne. En effet, dans $57 \%$ des relevés la circonférence moyenne des arbres est inférieure à $10 \mathrm{~cm}$ (fig 4). Le reste de la populationéchantillon est approximativement divisé en 3 groupes d'arbres dont la circonférence moyenne est comprise entre 10 et $25 \mathrm{~cm}(17 \%)$, entre 25 et $40 \mathrm{~cm}(14 \%)$ et des arbres dont la circonférence est supérieure à $45 \mathrm{~cm}(12 \%)$.

Pour réaliser la cartographie des circonférences moyennes et des densités, les valeurs des circonférences sont réparties en 5 classes et sont symbolisées par des cercles de diamètre variable. Les densités sont également divisées en 5 classes, 


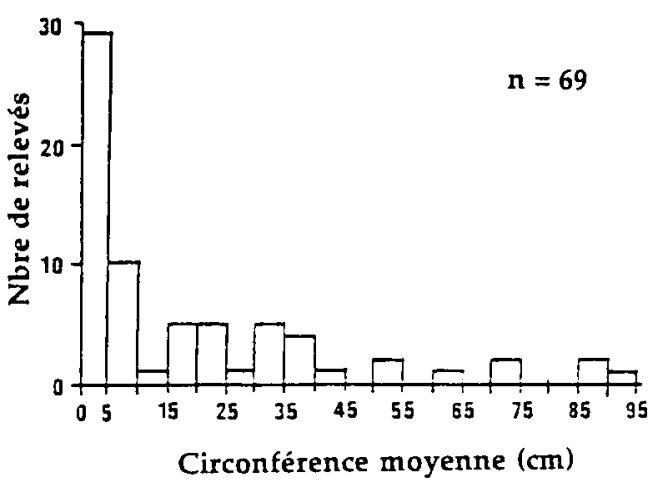

Fig 4. Distribution des circonférences moyennes de la population échantillon (calculées à partir de 4 individus.

symbolisées par des nuances de gris (fig 5).

Globalement, la circonférence moyenne des aulnes diminue de l'ouest vers l'est du secteur d'étude et la densité augmente. Cependant, plusieurs zones de discontinuité sont visibles. D'une part entre le premier et le deuxième tiers du secteur en partant de l'ouest, et d'autre part, au niveau du pare-feu.
Dans le pare-feu, les aulnes ont une circonférence moyenne inférieure à $5 \mathrm{~cm}$ et la densité est de 10-30 arbres par $100 \mathrm{~m}^{2}$, alors que dans la partie est de l'enclos, pour une circonférence moyenne équivalente, la densité est nettement supérieure : 50-100 aulnes/100 $\mathrm{m}^{2}$.

La plupart des aulnes mesurés dans le pare-feu sont en fait des rejets de souche. La densité observée correspondrait donc à celle de la population d'aulnes présente avant la fauche. D'autre part, la fauche a favorisé le développement de Phragmites australis, ralentissant ainsi la progression de l'aulne.

\section{Deuxième série de mesures}

Les 3 premiers facteurs de l'analyse des correspondances ont été retenus pour l'interprétation des résultats, puisqu'ils expriment à eux trois $97 \%$ de la variation interrelevés. Pour chaque facteur, les coordonnées factorielles des colonnes (classes de circonférence) sont représentées comme fonction de la valeur centrale de la classe de circonférence. Ce type de graphique est appelé : représentation fonc-

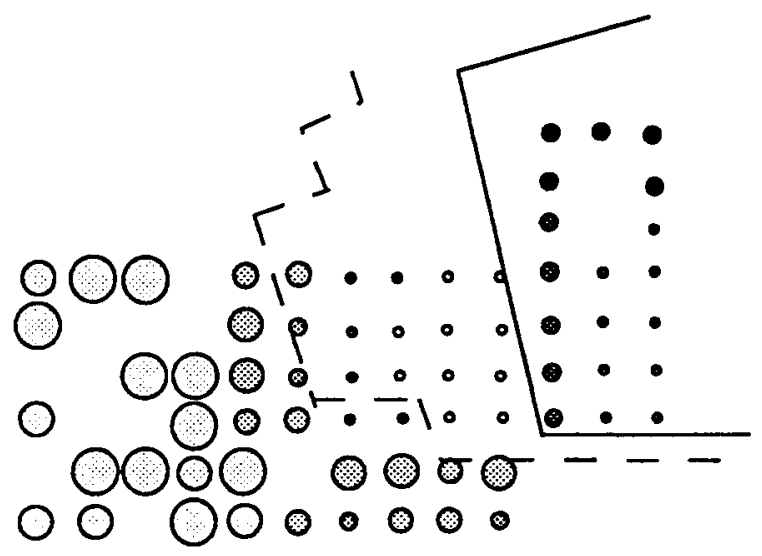

Circonferences :

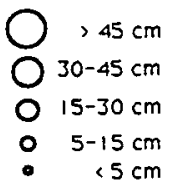

Densite (arbres/ $100 \mathrm{~m} 2)$ :

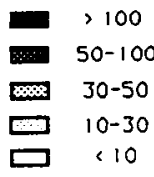

Fig 5. Cartographie des circonférences moyennes des aulnes $(\mathrm{cm})$ et des densités (nb arbres/ $100 \mathrm{~m}^{2}$ ) dans le secteur échantillonné. 
tionnelle des facteurs. «Des variations lentes des coordonnées factorielles indiquent de larges chevauchements des histogrammes, tandis que des variations brutales entre 2 classes de circonférence contiguës mettent en évidence des groupes d'histogrammes non chevauchants" (Persat et Chessel, 1989). Les coordonnées factorielles des lignes (relevés) sont cartographiées sur le plan du secteur étudié, sous forme de carrés de taille proportionnelle à la valeur absolue de la coordonnée et à l'intérieur desquels on note son signe. La lecture simultanée de ces 2 représentations aide à l'interprétation des résultats.

Le premier facteur de l'analyse des correspondances fait apparaître 2 groupes de classes de circonférence (fig 6a F1) : l'un, constitué par les petites classes de circonférence ordonnancées selon un gradient, et l'autre, constitué par les classes de circonférence $>10 \mathrm{~cm}$. La structure graduelle des coordonnées factorielles dans le premier groupe signifie que les histogrammes de distribution des circonférences associés à chaque relevé ne se chevauchent pas ou très peu. Par contre, il est plus difficile de distinguer une classe dominante dans les relevés comprenant des arbres de circonférence $>10 \mathrm{~cm}$, au vu de l'homogénéité des coordonnées factorielies du deuxième groupe. De même, la cartographie des coordonnées factorielles des lignes met en évidence 2 groupes de relevés : la partie ouest de la population composée des arbres les plus gros et la partie est qui correspond à la zone de colonisation récente (fig $6 \mathrm{~b} F 1$ ).

Le deuxième facteur de l'analyse sépare les classes de circonférence comprises entre 10 et $40 \mathrm{~cm}$ de l'ensemble des 12 dernières classes (fig 6a F2).

Le facteur 3 confirme la partition obtenue avec le facteur 2 tout en apportant une nouvelle information sur la répartition spa- tiale des circonférences au niveau du pare-feu. Les relevés situés autour de la zone fauchée ont des coordonnées factorielles de valeurs relativement proches. Ils appartiennent probablement à un même groupe qui se trouvait à la place du parefeu avant la fauche.

D'après les résultats de l'analyse factorielle des correspondances, la population étudiée semble structurée en 4 groupes principaux. En bordure ouest du secteur, le long de la rivière, se trouvent les arbres les plus gros, correspondant à la population initiale à partir de laquelle le marais a été colonisé. Cette souspopulation est constituée d'arbres ayant une circonférence $>40 \mathrm{~cm}$. L'hétérogénéité des circonférences dans cette zone est probablement liée à l'effet de la compétition interindividuelle et aux régénérations qui ont pu se faire dans les trouées d'origine naturelle ou artificielle (Harper, 1977). Trois autres groupes se succèdent dans l'espace, selon un gradient de circonférences décroissantes, le pare-feu mis à part (fig 6c F3). Ils regroupent des relevés ayant des classes de circonférence dominantes proches et s'étendent sur 100-150 $\mathrm{m}$ en direction de l'est. Comme les valeurs des circonférences sont relativement homogènes dans chaque groupe, on peut supposer qu'ils constituent chacun une vague de colonisation.

Sachant que l'aulne atteint sa maturité vers 10 ans et que la distance moyenne de dissémination des akènes est de $60 \mathrm{~m}$, voire $100 \mathrm{~m}$, sa vitesse de progression serait alors de l'ordre d'une centaine de mètres en une dizaine d'années (Mac Vean, 1953, 1955a,b). Nous avons vérifié cette hypothèse en comparant les photographies aériennes du secteur prises en $1939,1971,1980$ et 1985 et la carte (fig 6c F3) obtenue avec le facteur 3 de l'AFC. La limite ouest du groupe 1 coincide avec le front de progression de la forêt (essentiellement constituée d'aulnes à cet endroit) 
a)

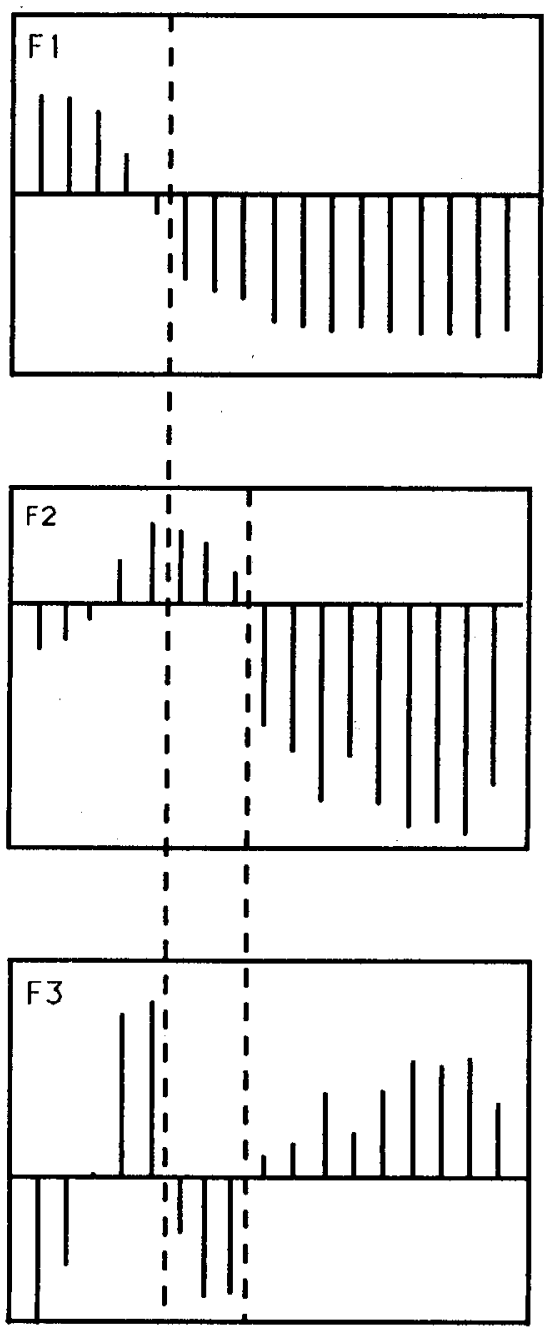

b)
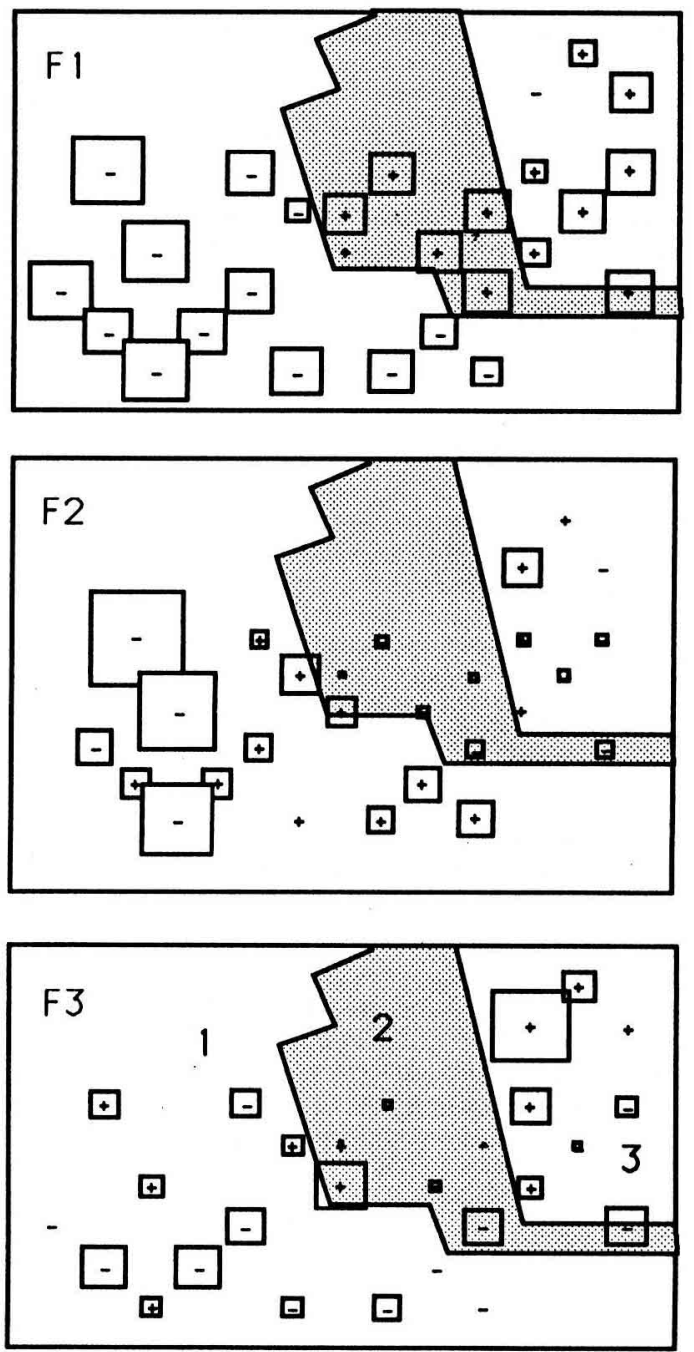

1234567891011121314151617

Fig 6. Résultats de l'analyse factorielle des correspondances des 27 relevés de la $2 e$ série de mesures. a) Représentation fonctionnelle des coordonnées factorielles des classes de taille sur les 3 premiers facteurs de l'analyse (F1, F2 et F3). De 1 à 4 la taille des aulnes est exprimée par leur hauteur en $\mathrm{cm}$, leur circonférence est inférieure à $5 \mathrm{~cm}(1: 0-50 ; 2: 50-80 ; 3: 80-200 ; 4:>200)$, de 5 à 17 par leur circonférence en $\mathrm{cm}(5: 5-10 ; 6: 10-20 ; 7: 20-30 ; 8: 30-40 ; 9: 40-50 ; 10: 50-60 ; 11$ : $60-70 ; 12: 70-8 ; 13: 80-90 ; 14: 90-100 ; 15: 100-110 ; 16: 110-120 ; 17:>120$ ). b) Représentation spatiale par carrés des coordonnées factorielles des relevés sur fond de carte du secteur. Le parefeu apparaît en grisé. 
en 1939, sa limite est, avec le front de progression de la forêt en 1971.

La première vague de colonisation (groupe 1) s'est donc mise en place en une trentaine d'années. En ce qui concerne la deuxième vague de colonisation (groupe 2), il a fallu environ 15 ans (de 1971 à 1985) pour que l'aulne s'installe. La progression de l'aulne a été plus rapide lors de la deuxième vague de colonisation. La partie ouest du secteur d'étude, jusqu'à l'enclos, se trouve dans la zone privée du marais (par opposition au marais communal). Les dernières parutions des bans de fauchaison concernant les parcelles privées datent de juillet-août 1928, mais certains propriétaires fauchaient encore il y a une vingtaine d'années, ce qui a du ralentir l'extension de l'aulne.

Les groupes individualisés par l'AFC semblent donc bien correspondre à des vagues successives de colonisation. II serait cependant intéressant de mesurer la vitesse de progression de l'aulne par une étude de la distribution spatiale de l'âge dans la population-échantillon, afin de valider cette méthode et de l'utiliser pour cartographier la colonisation de l'aulne dans l'ensemble du marais.

\section{CONCLUSION}

L'analyse factorielle des correspondances réalisée sur les distributions des circonférences, s'est révélée un outil intéressant pour étudier l'extension de l'aulne. Nous avons pu mettre en évidence le schéma de colonisation des arbres à partir d'une mesure simple.

Cette méthode n'est cependant valable que lorsque la relation âge-circonférence est forte, ce qui est le cas pour les populations jeunes. Avec le temps, les effets de la compétition interindividuelle s'accentuent et cette correspondance tend à s'es- tomper. Pour une étude plus précise, il serait nécessaire d'établir une relation entre l'âge et la circonférence par régression à partir d'un échantillon.

Dans cette étude, nous avons considéré l'extension de l'aulne en fonction de facteurs uniquement intrinsèques. Or si la vitesse de colonisation de l'auline dépend effectivement de ses capacités de dissémination elle est également fonction d'un ensemble de facteurs environnementaux qui favorisent ou empêchent l'arrivée des graines au sol, la germination et l'installation des plantules (Mac Vean, 1955a, 1959; Grime, 1979).

En vue d'appliquer cette méthode à l'ensemble du marais de Lavours, il serait donc indispensable de tenir compte de l'hétérogénéité des conditions stationnelles, liée en particulier à l'alimentation en eau et à la nature des sols en fonction de la proximité des cours d'eau principaux (le Rhône et le Séran) (Sogreah, 1960; Ain et Pautou, 1969).

\section{REMERCIEMENTS}

Je tiens à remercier toutes les personnes qui m'ont aidée à réaliser ce travail, et plus particulièrement :

- M Pautou pour son accueil dans son laboratoire;

- M Chessel pour ses conseils dans le traitement des données;

- M Reygrobellet pour ses critiques et suggestions;

- l'entente interdépartementale pour la démoustication de Chindrieux (Savoie) pour sa collaboration technique sur le terrain.

\section{RÉFÉRENCES}

Aïn G, Pautou G (1969) Étude écologique du marais de Lavours (Ain). Documents pour la carte de la végétation des Alpes, Grenoble, VII : 25-64 
Bergeron $Y$, Dubuc M (1989) Succession in the southern part of the Canadian boreal forest. Vegetatio 79, 51-63

Bravard JP (1987) Le Rhône : du Léman à Lyon. La Manufacture, Lyon, $451 \mathrm{p}$

Cottam G, Curtis JT (1956) The use of distance measures in phytosociological sampling. Ecology 37, 451-460

Gounot M (1969) Méthodes d'étude quantitative de la végétation. Paris, Masson, $314 \mathrm{p}$

Grime JP (1979) Plant strategies and vegetation processes. J Wiley and Sons, $210 \mathrm{p}$

Harper JL (1977) Population biology of plants. Acad Press, $891 \mathrm{p}$

Lhôte $P$ (1985) Étude écologique des Aulnes dans leur aire naturelle en France. Rapport IDF, ENGREF, Univ Franche-Comté Besançon, $67 \mathrm{p}+$ annexes

McVean DN (1953) Account of Alnus glutinosa (L) Gaertn (for the Biological Flora of the British isles). J Ecol 41, 447-466

McVean DN (1955a) Ecology of Alnus glutinosa (L) Gaertn. I. Fruit formation. J Ecol 43, 46-60

McVean DN (1955b) Ecology of Alnus glutinosa (L) Gaertn. II. Seed germination and distribution. $J$ Ecol $43,61-71$

McVean DN (1959) Ecology of Alnus glutinosa (L) Gaertn. VII. Establishment of alder by direct seeding of shallow blanket bog. J Ecol 47, 615-618

Manneville O, Majchrzak $Y$ (1988) Types de cartographie du couvert végétal dans le suivi de la gestion du pâturage d'une réserve naturelle (marais de Lavours, Ain, France). Doc Cartog Ecol XXXI, Univ Grenoble, 61-72

Olsson EG (1987) Effects of dispersal mechanisms on the initial pattern of olfield forest succession. Acta Oecol Oecol Gen 8, 379-390

Pautou $G$ (1980) La dynamique de la végétation dans la vallée du Rhône entre Genève et Lyon (J Cramer, ed) Coll Phytosoc IX, Les Forêts alluviales, 81-91

Pautou G (1985) Problèmes biologiques posés par l'extension des peuplements de ligneux dans le marais de Lavours. Publication de l'entente interdépartementale pour la dé- moustication (Chindrieux-Savoie) et de l'Univ de Grenoble, 19 p

Pautou G, Decamps H (1985) Ecological interactions between the alluvial forests and hydrology of the upper Rhone. Arch Hydrobiol 104, 13-37

Pautou G, Girel J, Lachet B, Ain G (1979) Recherches écologiques dans la vallée du Haut-Rhône français. Doc Cartog Ecol XXII, Univ Grenoble, 5-63

Pautou G, Decamps H, Amoros C, Bravard JP (1985a) Successions végétales dans les couloirs fluviaux : l'exemple de la plaine alluviale du Haut-Rhōne français. Bull Ecol 16, 203212

Pautou G, Girel J, Maman L (1985b) Le rôle des processus allogéniques dans le déroulement des successions végétales: l'exemple de la plaine alluviale du Rhône entre Genève et Lyon. In: Coll Phyosoc XIII, Végétation et géomorphologie, Bailleul, 655-668

Persat H, Chessel D (1989) Typologie de distributions en classes de taille : intérêt dans l'étude des populations de poissons et d'invertébrés. Acta Oecol Oecol Gener 10, 175195

Pielou EC (1959) The use of point-to-point plant distances in the study of the pattern of plant populations. J Ecol $47,607-613$

Roux AL, Amoros C, Richardot-Coulet M, Reygrobellet JL (1982) Cartographie polythématique appliquée à la gestion écologique des eaux. Étude d'un hydrosystème fluvial : le Haut-Rhône français. PIREN, CNRS, $113 \mathrm{p}$, 2 cartes

SOGREAH (Sociétė grenobloise d'études et d'applications hydrauliques) (1960) Mise en valeur des marais de Lavours. Étude agronomique, Grenoble, 85 p

Thioulouse J (1989) Statistical analysis and graphical display of multivariate data on Macintosh. Comput Appl Biosc 5, 287-292

Vinther E (1983) Invasion of Alnus glutinosa (L) Gaertn in a former grazed meadow in relation to different grazing intensities. Biol Consen $25,75-89$ 